\title{
Effectiveness of screening for oral cancer not proven
}

\author{
Are screening programmes effective in reducing mortality rates from oral \\ cancer?
}

\author{
Kujan O, Glenny AM, Duxbury AJ, Thakker N, Sloan P. Screening \\ programmes for the early detection and prevention of oral cancer \\ (Cochrane Review). Cochrane Library. Chichester: John Wiley; \\ 2003, Issue 4
}

Data sources Electronic databases (Medline, Cancerlit, Embase, the Cochrane Central Register of Controlled Trials) from 1966 to September 2002 and the Cochrane Library up to issue 2 in 2002 were searched. Searches by hand of specific journals and bibliographies were also made and corresponding authors contacted to identify published and unpublished work.

Study selection Studies were required to be randomised controlled trials (RCT) that screened for oral cancer or potentially premalignant oral lesions using visual examination, toluidine blue, fluorescence imaging or brush biopsy.

Data extraction and synthesis Data were extracted independently by two reviewers.

Results Only one RCT met the review's inclusion criteria. This one ongoing trial has 13 clusters of 153708 eligible subjects and 130799 included subjects. There was no difference in the age-standardised oral cancer mortality rates for the screened group (21.2/100 000 personyears) and the control group (21.3/100000 person-years). This study has some methodological weaknesses, however.

Conclusions Given the limited evidence (only one RCT) and the potential methodological weakness in that study, it is not possible to say whether there is evidence to support or refute the use of a visual examination as a method of screening for oral cancer in the general population. Furthermore, no robust evidence exists to suggest whether other methods of screening - toluidine blue, fluorescence imaging or brush biopsy - are either beneficial or harmful. Further cost-effective, high-quality studies to assess the efficacy and effectiveness of screening are required. In addition, studies to elucidate the natural history of oral cancer, prevention methods and the effectiveness of opportunistic screening in high-risk groups are needed.

Address for correspondence: Emma Tavender, Review Group Co-ordinator, Cochrane Oral Health Group, University Dental Hospital of Manchester, Higher Cambridge Street, Manchester M15 6FH, UK. E-mail: emma.tavender@man.ac.uk.

\section{Commentary}

This Cochrane review was performed with the primary goal of assessing the effectiveness of current screening programmes in reducing mortality due to oral cancer. As with many Cochrane reviews, the end product suffers because of the lack of high-quality research investigating the subject matter relevant to the review. The authors observe that systematic reviews of oral cancer screening programmes in general, ${ }^{1}$ and of the performance of visual exams ${ }^{2}$ and toluidine blue ${ }^{3}$ in particular, have recently been performed. The collective conclusions from these reports were that there was insufficient evidence to support screening programmes for oral cancer, ${ }^{1}$ that visual examination had fair sensitivity (but with considerable heterogeneity in the results, 0.60-0.95) and very good specificity (0.94-0.99), ${ }^{2}$ and that toluidine blue provided no benefit over the visual examination when screening for oral cancer. ${ }^{3}$ The authors of this Cochrane review also point out that the aforementioned systematic reviews had broader, or no, inclusion and exclusion criteria for the studies identified. In contrast, the current Cochrane review imposed a scientifically strong criterion but, unfortunately, this resulted in the authors including only one, ongoing, RCT. They were therefore unable to add anything new to our current knowledge of the effectiveness of screening for oral cancer.

The review itself obviously focuses largely on this one study, the Trivandrum Oral Cancer Screening Study, in Kerala, India. The sample includes 130779 adults (35 years of age or older) who are residents of Trivandrum. They are divided into 13 clusters according to rural areas, and randomised into seven areas whose residents are receiving the screening intervention and six areas whose residents are not being screened for oral cancer. Details of the study can be found in the two articles reporting the work that have so far been publised. ${ }^{4,5}$ Suffice to say that, as reported in the abstract of the Cochrane review, several years into this ongoing project no differences in oral cancer-related mortality rates have been found between the test and control groups. It also important to recognise that, whatever the final outcome of this study, the ability to generalise from its findings will be limited by the nature of the intervention and the study setting.

Beyond this core to the review, the background introductory material and the methodology raised a number of issues. The authors provide a brief background literature overview to justify the performance of the review, although it is somewhat disappointing to note that, when describing oral cancer incidence, they use secondary reference sources rather than cancer registry data. Furthermore, the authors use the oft-quoted 50\% 5-year survival rate for oral cancer. Although this figure may suffice for a very crude indication of oral cancer survival, there is enormous variation by country, anatomic site, gender and age, among other variables, and it would be useful for a systematic review to qualify the crude figure with these clarifications. Finally, the authors mention the classic definition of 'oral cancer' in terms of the ICD (International Classification of Diseases) classifications but fail to acknowledge the differences between the various oral cancers. This is important as the 
generic term 'oral cancer' often includes cancers at sites as different as the lip, salivary glands, tongue and nasopharynx. The epidemiology, aetiology, pathology and natural history of these cancers are often very different and this needs to be considered when designing research and making inferences concerning oral cancer. Furthermore, it is fundamental to the screening criteria set out by Wilson and Jungner ${ }^{6}$ that the natural history and speed of onset of the disease to be screened must be understood, so that screening intervals may be set. These criteria are not met for the most common form of oral cancer, squamous cell carcinoma of the mucosa of the mouth, tongue and oropharynx, but they are certainly different to, for instance, the natural history of salivary gland malignancies. Grouping salivary gland mali gnancies with the more common oral cancers such as those of the tongue, floor of mouth and oropharynx, is similar to grouping breast and lung cancers or colon and prostate cancers together because of their anatomic proximity. This does not mean that it cannot be done but we need to understand the implications of doing this.

Following this brief background review, the authors describe the review methodology in the traditional manner for this sort of work. They restricted studies to be included to those with a RCT design only. The types of intervention included were, 'Any oral screening programme for the early detection of oral cancer or potentially premalignant oral lesions'. These screening programmes could include visual examinations, the use of toluidine blue, fluorescence imaging and brush biopsy. Unfortunately, the description of included interventions did not make it clear whether the screening programmes were specific to oral cancer or if routine dental examinations (of the 6-month check-up type) were also included. When looking at the bibliography, one presumes that the routine dental examination was not included.

The authors described their primary outcome as oral cancerspecific mortality but they were also interested in secondary outcomes such as disease stage at diagnosis, quality of life and cost. They provided a copy of the search strategy, which included the classic databases and the majority of keywords one would have expected, although here again, the definition of oral cancer is not clear because the keywords presented included, among others, 'lip' and 'hypopharynx' but did not include 'floor of mouth', 'salivary glands' and 'nasopharynx'.

In conclusion, this review, through no fault of its own, unfortunately adds nothing to our understanding of the effectiveness of screening for oral cancer but makes it very clear that more well-designed research is necessary on this subject.

\section{Paul Allison}

Faculty of Dentistry, McGill University, Montreal, Quebec, Canada

1. Hawkins RJ, Wang EE, Leake JL. Preventive health care, 1999 update: prevention of oral cancer mortality. The Canadian Task Force on Preventive Health Care. J Can Dental Assoc 1999; 65:617.

2. Moles DR, Downer MC, Speight PM. Meta-analysis of measures of performance reported in oral cancer and precancer screening studies. Br Dental J 2002; 192:340-344

3. Gray M, Gold L, Elley A, Bursla A. The Effectiveness of Toluidine Blue Dye as Adjunct to Oral Cancer Screening in General Dental Practice. Birmingham: University of Birmingham Department of Public Health and Epidemiology; 2000.

4. Sankaranarayanan R, Mathew B, Jacob BJ, et al. Early findings from a communitybased, cluster-randomized, controlled oral cancer screening trial in Kerala, India. Cancer 2000; 88:664-673.

5. Ramadas K, Sankaranarayanan R, Jacob BJ, et al. Interim results from a cluster randomized controlled oral cancer screening trial in Kerala, India. Oral Oncol 2003, 39:580-588.

6. Wilson JMG, Jungner G. Principles and Practice of Screening for Disease. Geneva WHO; 1968; (Public health paper No 34.)

Evidence-Based Dentistry (2004) 5, 40-41.

doi:10.1038/sj.ebd.6400252 